\title{
Editorial: Major to Great Earthquakes: Multidisciplinary Geophysical Analyses for Source Characterization
}

\author{
Debora Presti ${ }^{1 *}$, Sebastiano D'Amico ${ }^{2}$ and loannis Kassaras ${ }^{3}$ \\ ${ }^{1}$ Department of Mathematics, Computer Science, Physics, and Earth Sciences, University of Messina, Messina, Italy, \\ ${ }^{2}$ Department of Geosciences, University of Malta, Msida, Malta, ${ }^{3}$ Department of Geology and Geoenvironment, National and \\ Kapodistrian University of Athens, Athens, Greece
}

Keywords: major to great earthquakes, geophysical inversion methods, physical and numerical modelling, instrumental data, seismogenic sources

\section{Editorial on the Research Topic}

Major to Great Earthquakes: Multidisciplinary Geophysical Analyses for Source Characterization

In recent years, the study of Major to Great earthquakes is strongly supported by the increase of technological and scientific capabilities such as dense global networks of seismic sensors, GPS stations and satellite imaging, tsunami gauges, geophysical surveying of the gravity of the Earth and more and more accurate techniques of data analysis. This enhancement in earthquake research facilitates unprecedented analyses of precursory, co-seismic, post-seismic processes, as well as their impact through comprehensive modelling of their consequences. Nevertheless, there are cases that information for significant earthquakes like location, moment tensor, rupture propagation, relation with secondary effects are to date poorly known and both the causative sources and triggering forces are still debated. Such ambiguities are often the source of misleading seismic hazard estimates that may result in unexpected adverse impacts.

The main goal of this Research Topic was to assemble studies of seismogenic sources able to produce major to great earthquakes, mainly focusing on their nature and rupture properties, interactions with nearby sources, spatiotemporal arrangement of aftershocks, and induced secondary effects. The works published herein are based on the application of various geophysical techniques such as inversion methods, processing of seismological, geodetic and gravimetric data, remote sensing, physical and numerical modelling. We believe that the overall effort, apart from shedding light at active regions of interest could provide a multidisciplinary framework for future research as well.

The mini review by Funiciello et al., presents the current state of knowledge achieved from application of empirical analysis of global-scale natural data and experimental data from analogue modelling to the study of cause-effect relationships between subduction zone parameters and the megathrust seismogenic behavior. The combination of the two approaches allows drawing conclusions about the geodynamic conditions that may favor the occurrence of giant megathrust earthquakes such as the Indonesia 2004, Chile 2010, and Japan 2011 earthquakes.

During the last 2 decades, space geodesy allowed mapping accurately rupture areas, slip distribution, and seismic coupling by obtaining refined inversion models and greatly improving the study of great megathrust earthquakes. Álvarez et al., by performing direct modeling of satellite GOCE, analyzed seismic rupture behavior for recent and for historical earthquakes along the Sunda subduction margin and the relationship of the degree of interseismic coupling.

The study by Singh and Khan, deals with the computations and mapping of the basement configuration, sediment thickness, Moho depth, and residual isostatic gravity anomaly, in the eastern 
Indian shield and adjoining regions. Main findings were interpreted in terms of stress field distribution generating great earthquakes in the Nepal-Bihar-Sikkim Himalaya, and causing deformation and seismicity in the adjacent areas.

Romano et al., investigated the source model of the 2010 Chile, Mw 8.8, earthquake by benchmark the method of Optimal Time Alignment of the tsunami waveforms in the joint inversion of tsunami (DART and tide-gauges) and geodetic (GPS, InSAR, land-leveling) observations for this event. The results obtained show that the first-order modeling correction introduced by OPT confirms the bilateral rupture pattern around the epicenter and shifts the inferred northern patch of slip to a shallower position consistent with the slip models obtained by applying more complex physics-based corrections to the tsunami waveforms.

The subject of rupture mechanism of deep-focus $(>300 \mathrm{~km})$ earthquakes in subducting slabs of oceanic lithosphere is addressed by Zhang et al. The rupture model for the 2013, Mw 8.3, deep Okhotsk Sea earthquake, is constrained by a multi-array and multi-frequency combined back-projection method as well as a global P-wave train inversion technique for discrete subevents. The earthquake is found to rupture at different speeds to the north and to the south, respectively, of the epicenter. This evidence newly supports dehydration embrittlement as a viable and possible unifying mechanism for the majority of deep earthquakes.

A physics-based earthquake simulator is implemented by Kourouklas et al. in the Southern Thessaly Fault Zone (Greece), aiming to provide insights about the recurrence behavior of earthquakes with $\mathrm{Mw} \geq 6.0$ in six major fault segments in the study area. The application of the algorithm produced a seismic catalog for $10 \mathrm{kyr}$ containing 254 events with $\mathrm{Mw} \geq 6.0$ implying both single and multi-segment ruptures. The large number of simulated ruptures provides the ability of a thorough investigation of their recurrence properties.

Major seismicity of southern Italy is analyzed in four contributions. Bello et al., use various geological and revised seismological data in order to reconstruct the surface and depth geometry, the kinematics and stress tensor related to the Campania-Lucania 1980 earthquake (Mw 6.9). Three main fault alignments are identified. Strain and stress analyses show NNE extension. The proposed seismotectonic model includes a deep graben-like structure.

Neri et al. (a) find that very strong shallow seismicity of southern Calabria, imputed to normal faulting, corresponds to relatively low values of local, geodetic horizontal strain rate, and note that this situation apparently contrasts with observations in the Apennines, from central Italy to northern Calabria, where normal faulting earthquake activity of lower magnitude corresponds to relatively large values of extensional strain rate. The authors suggest a possible explanation of this somewhat unusual situation.

A detailed study of the Messina Straits area is proposed by Neri et al. (b), based on high-quality hypocenter locations and waveform inversion focal mechanisms of recent earthquakes. Seismicity in the area of the major, 1908 earthquake has mainly occurred below the east-dipping north-striking fault proposed by most investigators as the source of the 1908 earthquake, while it has been substantially absent in correspondence of the fault and above it. This distribution of seismicity suggests the existence of a huge, low-fractured shallow block resting on a somewhat fractured medium, with the separation surface between them corresponding to the 1908 earthquake fault.

Finally, marine seismic reflection data coupled with on-land structural measurements allowed Gambino et al. to achieve new information about active deformation pattern of the northern sector of the Malta Escarpment, a bathymetric and structural discontinuity capable of major earthquakes in the near-offshore of Eastern Sicily. Dimensional parameters of detected faults were then used to estimate expected magnitudes and their reactivation propensity.

\section{AUTHOR CONTRIBUTIONS}

All authors listed have made a substantial, direct, and intellectual contribution to the work and approved it for publication.

Conflict of Interest: The authors declare that the research was conducted in the absence of any commercial or financial relationships that could be construed as a potential conflict of interest.

Publisher's Note: All claims expressed in this article are solely those of the authors and do not necessarily represent those of their affiliated organizations, or those of the publisher, the editors and the reviewers. Any product that may be evaluated in this article, or claim that may be made by its manufacturer, is not guaranteed or endorsed by the publisher.

Copyright (c) 2021 Presti, D'Amico and Kassaras. This is an open-access article distributed under the terms of the Creative Commons Attribution License (CC BY). The use, distribution or reproduction in other forums is permitted, provided the original author(s) and the copyright owner(s) are credited and that the original publication in this journal is cited, in accordance with accepted academic practice. No use, distribution or reproduction is permitted which does not comply with these terms. 